
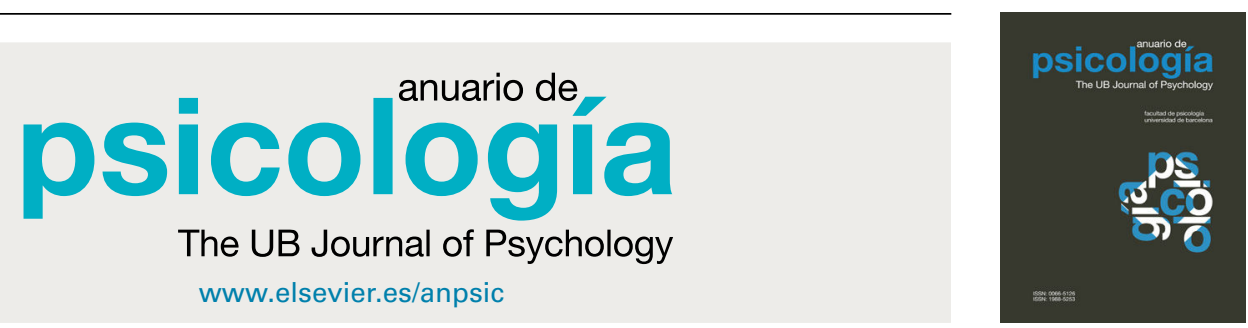

ARTÍCULO DE INVESTIGACIÓN

\title{
Estresores académicos percibidos por estudiantes universitarios y su relación con el burnout y el rendimiento académicos
}

\section{Carmen María Vizoso Gómez* y Olga Arias Gundín}

Departamento de Psicología, Sociología y Filosofía, Universidad de León, León, España

Recibido el 16 de febrero de 2016; aceptado el 27 de junio de 2016

\author{
PALABRAS CLAVE \\ Estresores \\ académicos; \\ Burnout académico; \\ Rendimiento \\ académico; \\ Eficacia académica; \\ Estudiantes \\ universitarios
}

\begin{abstract}
Resumen El objetivo de este estudio es conocer las principales fuentes de estrés percibidas por los estudiantes universitarios en el contexto académico y analizar las relaciones de estos estresores con el burnout académico y el rendimiento. Participaron 532 estudiantes de la Universidad de León. Los instrumentos empleados fueron la Escala de Estresores Académicos y la versión española del Maslach Burnout Inventory-Student Survey. Los resultados revelan que las situaciones relacionadas con los exámenes son las percibidas como estresantes con más frecuencia. La carencia de valor de los contenidos predice significativamente todas las dimensiones del burnout. Además, la eficacia y el rendimiento se predicen por la preocupación por las deficiencias metodológicas y por la falta de participación. Por otra parte, la preocupación por las intervenciones en público afecta negativamente tanto al rendimiento como a la eficacia. Con el fin de reducir el estrés y el burnout y favorecer el rendimiento de los estudiantes se consideran posibles medidas de intervención.

(c) 2016 Universitat de Barcelona. Publicado por Elsevier España, S.L.U. Todos los derechos reservados.
\end{abstract}

\section{Academic stressors perceived by university students and their relationship with academic burnout, efficacy and performance}

\begin{abstract}
This study aimed to examine the principal stress sources perceived by university students at academic context and to analyze the relationship between these stressors and academic burnout and performance. Participants were 532 undergraduate students from Universidad de León. The techniques used in this survey were Academic Stress Questionnaire and the Spanish version of the Maslach Burnout Inventory-Student Survey. Results showed that situations related to exams are perceived as stressor more frequently. Lack of value from the contents is a significant predictor of burnout. In addition, efficacy and performance are predicted by
\end{abstract}

\footnotetext{
* Autor para correspondencia.

Correo electrónico: cvizg@unileon.es (C.M. Vizoso Gómez).
} 
teacher methodological shortcomings and lack of participation. On the other hand, stress caused by public participation shown a negative influence on both efficacy and performance. To reduce stress level and burnout and improve students' performance, possible intervention strategies are proposed.

(c) 2016 Universitat de Barcelona. Published by Elsevier España, S.L.U. All rights reserved.

\section{Introducción}

El estrés surge cuando un individuo percibe que una situación es amenazante o que desborda sus recursos para hacerle frente (Lazarus, 2000). Por ello, se puede experimentar estrés en cualquier contexto ante situaciones adversas que se dan en la vida cotidiana (Kanner, Coyne, Schaefer y Lazarus, 1981). Así, los estudiantes también pueden percibir como estresantes algunas de las situaciones que deben afrontar en el contexto académico (Arribas, 2013; Martín, 2007; Peñacoba y Moreno, 1999). En este sentido, Muñoz (2004) estableció que algunas circunstancias relativas a la vida académica, como las actividades, las demandas o los conflictos, pueden provocar estrés en los estudiantes e influir de forma negativa en su bienestar y en su rendimiento. Asimismo, especificó que las principales fuentes de estrés académico son los problemas de adaptación y transición dentro del sistema educativo, la sobrecarga de trabajo, la evaluación y las relaciones entre profesor y estudiante. Posteriormente, Arribas (2013) señaló que los estudiantes perciben como generadores de estrés los factores relacionados con la sobrecarga de tareas, la falta de tiempo para realizar el trabajo académico, los exámenes y evaluaciones y también, aunque en menor medida, la realización de trabajos obligatorios. En definitiva, concluyó que el estrés académico se concreta en 4 dimensiones: trabajos obligatorios, sobrecarga académica, percepción del profesor y percepción de la asignatura. Por otra parte, Cabanach, Souto, Freire y Ferradás (2014) evaluaron diferentes situaciones que los estudiantes percibían como estresantes y encontraron que los exámenes y las intervenciones en público eran las descritas como amenazantes con mayor frecuencia. Por el contrario, las situaciones que implican malas relaciones en el contexto académico y las relativas a la imposibilidad de participar en las decisiones que atañen al trabajo académico eran percibidas como estresoras con menor frecuencia (Cabanach, Fernández-Cervantes, González-Doniz y Freire, 2010; Cabanach, Rodríguez, Valle, Piñeiro y Millán, 2007; Cabanach et al., 2014; Cabanach, Valle, Rodríguez, Piñeiro y González, 2010).

De este modo, se ha confirmado la existencia de diferentes situaciones que generan estrés en los estudiantes universitarios. Teniendo en cuenta que en el ámbito laboral se defiende que los trabajadores sometidos a un estrés continuado «se queman» por su trabajo (Freudenberger, 1974; Maslach, 1976; Maslach, Schaufeli y Leiter, 2001), recientemente se ha planteado que los estudiantes universitarios pueden «quemarse» por sus estudios; es decir, pueden padecer burnout académico si perciben que deben afrontar condiciones estresantes frecuentemente y consideran que carecen de recursos para ello (Schaufeli, Martínez, MarquésPinto, Salanova y Bakker, 2002; Schaufeli y Salanova, 2007). Concretamente, se ha descrito que el burnout académico está caracterizado por altos niveles de cansancio emocional y de cinismo y por una baja eficacia respecto a las actividades académicas (Bresó, Schaufeli y Salanova, 2011; Manzano, 2002, 2004; Martínez, Marques, Salanova y Lopez da Silva, 2002; Schaufeli et al., 2002).

De forma general, existe un consenso en cuanto a la relación existente entre el estrés y el burnout académicos (Chambel y Curral, 2005; Muñoz, 2004). Asimismo, se ha establecido que el estrés académico suele relacionarse con situaciones de evaluación académica y de sobrecarga de trabajo (Arribas, 2013; Cabanach, Fernández-Cervantes et al., 2010; Cabanach, Valle et al., 2010; Cabanach et al., 2014). Por otra parte, se ha destacado que la eficacia y el rendimiento pueden estar relacionados con el estrés y el burnout (Chemers, Hu y Garcia, 2001; Muñoz, 2004; Salanova, Bresó y Schaufeli, 2005). Sin embargo, en la actualidad no existen estudios concluyentes donde se analicen las relaciones entre los factores citados: generadores de estrés, burnout y rendimiento.

En definitiva, el objetivo de este estudio es determinar cuáles son las situaciones percibidas como estresantes con más frecuencia y cuáles son las que se asocian con el burnout y/o con el rendimiento académicos para proponer medidas que capaciten a los estudiantes a afrontarlas con éxito y mejorar así su bienestar.

\section{Método}

\section{Participantes}

La muestra (de conveniencia) está formada por 532 estudiantes de diferentes titulaciones de la Universidad de León. La edad de los estudiantes oscila entre los 18 y los 52 años $(M=22,1 ; D T=2,19)$. Del total de la muestra, 438 estudiantes son mujeres y 94 son hombres, lo que supone una representación fiel de la distribución habitual por género en las titulaciones analizadas (Gratacós y López-Jurado, 2016; Manzanares y Martínez, 2014; Mendo-Lázaro, León, FelipeCastaño, Polo y Palacios-García, 2016). En la tabla 1 puede observarse la distribución de la muestra por titulación y curso. 
Tabla 1 Características de la muestra

\begin{tabular}{lcccrr}
\hline Grado & \multicolumn{3}{c}{ Curso } & Total \\
\cline { 2 - 5 } & Primero & Segundo & Tercero & Cuarto \\
\hline Educación Infantil & 61 & 65 & 74 & 58 & 258 \\
Educación Social & 31 & 35 & 39 & 30 & 135 \\
RRLL y RRHH & 33 & 22 & 44 & 40 & 139 \\
Total & 125 & 122 & 157 & 532 \\
\hline
\end{tabular}

RRLL y RRHH: Relaciones Laborales y Recursos Humanos.

\section{Instrumentos}

Para medir las situaciones que provocan estrés se empleó la Escala de Estresores Académicos (E-CEA), que forma parte del Cuestionario de Estrés Académico (Cabanach, Valle, Rodríguez y Piñeiro, 2008; Cabanach, Fernández-Cervantes et al., 2010). La escala está compuesta por 54 ítems, mediante los cuales se evalúan diferentes circunstancias del contexto académico que el estudiante puede percibir como amenazantes. Este instrumento se estructura en 9 factores, descritos en la tabla 2. La escala de respuesta es tipo Likert y oscila entre 1 («nunca») y 5 («siempre»). Los coeficientes de fiabilidad para los diferentes factores, medidos mediante el coeficiente alfa de Cronbach, oscilan entre 0,79 y 0,96 (Cabanach et al., 2007).
El burnout académico se evaluó mediante la versión española del Maslach Burnout Inventory-Student Survey (Schaufeli et al., 2002). Este instrumento está compuesto por 15 ítems y evalúa el grado en que el estudiante considera que está «quemado». Se estructura en 3 dimensiones: Cansancio emocional (por ejemplo, «Estoy cansado cuando me levanto por la mañana y tengo que afrontar otro día en la universidad»), Cinismo (por ejemplo, «Dudo de la trascendencia y valor de mis estudios») y Eficacia académica (por ejemplo, «Creo que contribuyo efectivamente durante las clases en la universidad»). La escala de respuesta es tipo Likert y oscila entre 0 («nunca») y 6 («siempre»). El burnout se identifica con altas puntuaciones en las dimensiones de cansancio y cinismo y bajas puntuaciones en la dimensión de eficacia. Los coeficientes de fiabilidad para los diferentes

Tabla 2 Factores de la Escala de Estresores Académicos

\begin{tabular}{|c|c|c|}
\hline Factor & Ítems & Ejemplo \\
\hline $\begin{array}{l}\text { 1. Deficiencias metodológicas } \\
\text { del profesorado }\end{array}$ & $10,11,12,13,14,15,16,17,18,19,20,21$ & $\begin{array}{l}\text { «Me pongo nervioso o me inquieto } \\
\text { cuando los profesores no se ponen } \\
\text { de acuerdo entre ellos» }\end{array}$ \\
\hline 2. Sobrecarga del estudiante & $27,29,31,32,33,34,36,38,39,40$ & $\begin{array}{l}\text { «Me pongo nervioso o me inquieto } \\
\text { por las demandas excesivas y } \\
\text { variadas que se me hacen» }\end{array}$ \\
\hline 3. Intervenciones en público & $1,2,3,4,9$ & $\begin{array}{l}\text { «Me pongo nervioso o me inquieto } \\
\text { cuando tengo que salir a la } \\
\text { pizarra» }\end{array}$ \\
\hline 4. Clima social negativo & $49,50,51,52,53,54$ & $\begin{array}{l}\text { «Me pongo nervioso o me inquieto } \\
\text { cuando hay favoritismos en clase» }\end{array}$ \\
\hline $\begin{array}{l}\text { 5. Falta de control sobre el } \\
\text { propio rendimiento } \\
\text { académico }\end{array}$ & $28,30,43,44,46$ & $\begin{array}{l}\text { «Me pongo nervioso o me inquieto } \\
\text { cuando no sé qué hacer para que } \\
\text { se reconozca mi esfuerzo y } \\
\text { trabajo» }\end{array}$ \\
\hline $\begin{array}{l}\text { 6. Carencia de valor de los } \\
\text { contenidos }\end{array}$ & $22,23,24,25$ & $\begin{array}{l}\text { «Me pongo nervioso o me inquieto } \\
\text { cuando lo que estoy estudiando } \\
\text { tiene una escasa utilidad futura» }\end{array}$ \\
\hline 7. Baja autoestima académica & $26,35,37,41,42$ & $\begin{array}{l}\text { «Me pongo nervioso o me inquieto } \\
\text { cuando no creo que pueda lograr } \\
\text { los objetivos propuestos» }\end{array}$ \\
\hline 8. Exámenes & $5,6,7,8$ & $\begin{array}{l}\text { «Me pongo nervioso o me inquieto } \\
\text { cuando preparo los exámenes» }\end{array}$ \\
\hline $\begin{array}{l}\text { 9. Falta de participación en el } \\
\text { propio trabajo académico }\end{array}$ & $45,47,48$ & $\begin{array}{l}\text { «Me pongo nervioso o me inquieto } \\
\text { porque no está en mi mano } \\
\text { plantear los trabajos, tareas o } \\
\text { actividades como me gustaría» }\end{array}$ \\
\hline
\end{tabular}


Tabla 3 Estadísticos descriptivos y correlaciones entre las fuentes de estrés

\begin{tabular}{|c|c|c|c|c|c|c|c|c|c|c|c|}
\hline Subescalas & M & DT & 1 & 2 & 3 & 4 & 5 & 6 & 7 & 8 & 9 \\
\hline 1 & 3,29 & 0,78 & - & & & & & & & & \\
\hline 2 & 3,11 & 0,68 & $0,732^{* *}$ & - & & & & & & & \\
\hline 3 & 3,22 & 1,16 & $0,263^{* *}$ & $0,363^{* *}$ & - & & & & & & \\
\hline 4 & 2,87 & 0,82 & $0,656^{* *}$ & $0,547^{* *}$ & $0,244^{* *}$ & - & & & & & \\
\hline 5 & 2,88 & 0,81 & $0,639^{* *}$ & $0,694^{* *}$ & $0,299^{* *}$ & $0,567^{* *}$ & - & & & & \\
\hline 6 & 2,94 & 0,88 & $0,618^{* *}$ & $0,437^{* *}$ & $0,117^{*}$ & $0,488^{* *}$ & $0,454^{* *}$ & - & & & \\
\hline 7 & 2,70 & 0,85 & $0,601^{* *}$ & $0,696^{* *}$ & $0,392^{* *}$ & $0,526^{* *}$ & $0,710^{* *}$ & $0,383^{* *}$ & - & & \\
\hline 8 & 3,36 & 0,93 & $0,424^{* *}$ & $0,641^{* *}$ & $0,493^{\text {*** }}$ & $0,361^{* *}$ & $0,487^{* *}$ & $0,165^{* *}$ & $0,580^{* *}$ & - & \\
\hline 9 & 2,85 & 0,847 & $0,561^{* *}$ & $0,437^{* *}$ & $0,150^{*}$ & $0,471^{\text {** }}$ & $0,438^{* *}$ & $0,512^{* *}$ & $0,315^{* *}$ & $0,270^{* *}$ & - \\
\hline
\end{tabular}

DT: desviación típica; $M$ : media.

1 = deficiencias metodológicas; 2 = sobrecarga; 3 = intervenciones en público; 4 = clima social negativo; 5 = falta de control sobre el rendimiento; 6 =carencia de valor de contenidos; 7 =baja autoestima académica; $8=$ exámenes; $9=$ falta de participación en el trabajo académico.

* $p<0,05$.

** $p<0,01$.

factores, medidos mediante el coeficiente alfa de Cronbach, oscilan entre 0,74 y 0,79 (Schaufeli et al., 2002).

Además, se registró el rendimiento académico de los estudiantes considerando la nota media del expediente académico hasta el momento de la aplicación de la prueba.

\section{Procedimiento}

Los datos se recogieron durante las primeras semanas del segundo semestre del curso por 2 razones: primero, porque es una época en la que no se realizan exámenes, eliminándose el posible estrés relacionado con la evaluación, y además, porque así los estudiantes de primer curso conocen los posibles estresores que conlleva realizar los estudios universitarios y pueden aportar la nota media correspondiente al primer semestre.

La aplicación de los cuestionarios se realizó en una sesión grupal en horario de clase de una asignatura de cada curso, contando con el consentimiento del profesor/a responsable. Los estudiantes participaron voluntariamente en el estudio tras ser informados sobre la intención de difundir los resultados de la investigación. Sistemáticamente, la sesión comenzaba con una explicación sobre el carácter confidencial y anónimo de la información aportada por los estudiantes. Además, durante toda la sesión, estaba presente una psicóloga experta para clarificar las dudas de los estudiantes.

\section{Análisis de datos}

Se empleó el programa estadístico SPSS, versión 20, para efectuar el análisis de datos. Para el cálculo de medias y desviaciones típicas se recurrió a la obtención de estadísticos descriptivos. Además, para determinar la fuerza y la dirección de las relaciones entre las diferentes fuentes de estrés y también entre las dimensiones de burnout y el rendimiento se recurrió al análisis de los índices de correlación (Pearson). Por último, se realizaron una serie de análisis de regresión para comprobar el valor predictivo de las distintas fuentes de estrés sobre los niveles de burnout y el rendimiento académico.

\section{Resultados}

En relación con los estresores académicos, en la tabla 3 se recogen los estadísticos descriptivos y las correlaciones entre los factores generadores de estrés. La situación percibida como estresante con más frecuencia es la relacionada con los exámenes, seguida de las deficiencias metodológicas del profesorado, las intervenciones en público y la sobrecarga del estudiante, todas ellas con medias superiores al valor de 3 sobre 5 . Por otra parte, la situación percibida como estresante con menor frecuencia es la baja autoestima académica. Además, los resultados mostraron que existe una relación estadísticamente significativa y positiva entre todas las situaciones.

En la tabla 4 se presentan los estadísticos descriptivos y las correlaciones entre las dimensiones del burnout y el rendimiento. Las relaciones entre las 3 dimensiones que definen al burnout fueron significativas. Específicamente, la relación entre cansancio y cinismo es positiva, mientras que la relación entre estas 2 dimensiones y la eficacia es negativa. El rendimiento se relaciona significativa y positivamente con la eficacia, pero negativamente con el cansancio. No existe relación entre rendimiento y cinismo.

La correlación entre las fuentes de estrés, el burnout y el rendimiento se recoge en la tabla 5 . Existe una relación significativa y positiva entre todas las fuentes de estrés y el cansancio; es decir, la percepción de estrés con mayor frecuencia en cualquiera de las situaciones se asocia con un mayor cansancio. Además, la mayoría de los estresores (excepto 2: intervenciones en público y exámenes) se relacionan también de forma significativa y positiva con el cinismo. Por último, las intervenciones en público y la baja autoestima académica se relacionan significativa y negativamente con la eficacia. Sin embargo, la falta de participación en el trabajo académico se asocia significativa y positivamente con la eficacia. Por otra parte, la falta de participación en el trabajo académico y las deficiencias metodológicas del profesorado correlacionan de forma 
Tabla 4 Estadísticos descriptivos y correlaciones entre burnout y rendimiento

\begin{tabular}{|c|c|c|c|c|c|c|c|}
\hline & & $M$ & DT & Cansancio & Cinismo & Eficacia & Rendimiento \\
\hline & Cansancio & 2,23 & 1,09 & - & & & \\
\hline \multirow[t]{2}{*}{ Burnout } & Cinismo & 1,76 & 1,12 & $0,588^{* *}$ & - & & \\
\hline & Eficacia & 4,02 & 0,84 & $-0,325^{* *}$ & $-0,358^{* *}$ & - & \\
\hline \multicolumn{2}{|c|}{ Rendimiento académico } & 6,90 & 0,78 & $-0,091^{*}$ & $-0,047$ & $0,398^{* *}$ & - \\
\hline
\end{tabular}

DT: desviación típica; $M$ : media.

* $p<0,05$.

${ }^{* *} \mathrm{p}<0,01$

Tabla 5 Correlaciones entre estresores, burnout y rendimiento

\begin{tabular}{|c|c|c|c|c|}
\hline Estresores & Cansancio & Cinismo & Eficacia & Rendimiento \\
\hline 1. Deficiencias metodológicas & $0,298^{* *}$ & $0,156^{* *}$ & 0,038 & $0,116^{* *}$ \\
\hline 2. Sobrecarga & $0,363^{* * *}$ & $0,093^{*}$ & $-0,022$ & $-0,010$ \\
\hline 3. Intervenciones en público & $0,160^{\text {** }}$ & $-0,013$ & $-0,130^{* *}$ & $-0,201^{* *}$ \\
\hline 4. Clima social negativo & $0,258^{* *}$ & $0,138^{* *}$ & 0,040 & 0,030 \\
\hline 5. Falta de control sobre el rendimiento & $0,304^{* *}$ & $0,156^{* *}$ & $-0,037$ & $-0,061$ \\
\hline 6. Carencia de valor de contenidos & $0,367^{* *}$ & $0,401^{* *}$ & $-0,078$ & $-0,143^{* *}$ \\
\hline 7. Baja autoestima académica & $0,295^{* *}$ & $0,109^{*}$ & $-0,146^{* *}$ & $-0,052$ \\
\hline 8. Exámenes & $0,231^{* *}$ & $-0,055$ & $-0,055$ & $-0,086$ \\
\hline 9. Falta de participación & $0,246^{* *}$ & $0,193^{* *}$ & $0,091^{*}$ & $0,130^{* *}$ \\
\hline
\end{tabular}

positiva con el rendimiento, mientras que las intervenciones en público y la carencia de valor de los contenidos lo hacen de forma negativa.

Los resultados de los análisis de regresión efectuados para determinar el valor predictivo de los estresores sobre los niveles de burnout y el rendimiento se presentan en la tabla 6.

En relación con las dimensiones de burnout, la carencia de valor de los contenidos predice de forma significativa los niveles de cansancio; es decir, cuanto mayor es la frecuencia de estrés por la carencia de valor, mayor es el nivel de cansancio. Respecto al cinismo, los estresores significativamente predictores son la carencia de valor y los exámenes. Concretamente, los estudiantes que perciben con frecuencia una carencia de valor y que muestran escasa preocupación por los exámenes presentan mayores niveles de cinismo. Por último, los estresores que predicen la eficacia son las deficiencias metodológicas, la falta de participación, las intervenciones en público, la baja autoestima académica y la carencia de valor de contenidos. Así, los estudiantes preocupados con mayor frecuencia por las deficiencias metodológicas y la falta de participación, pero que no suelen presentar estrés por intervenir en público, ni por una baja autoestima académica, ni tampoco por una carencia de valor, muestran mayor eficacia.

Finalmente, el rendimiento se predice por las deficiencias metodológicas, la falta de participación, las intervenciones en público y la falta de control sobre el propio rendimiento. Específicamente, los estudiantes preocupados con mayor frecuencia por las deficiencias metodológicas y la falta de participación, pero que casi nunca sienten estrés por realizar intervenciones en público o por percibir una falta de control sobre el rendimiento, muestran mejor rendimiento.

\section{Discusión}

En el presente estudio se analizaron las situaciones estresantes percibidas por los estudiantes universitarios y su relación con el burnout y el rendimiento académicos.

Los resultados obtenidos llevan a concluir que las situaciones relativas a los exámenes son las percibidas como estresantes con más frecuencia, en congruencia con lo descrito en investigaciones referidas al estrés académico (Cabanach et al., 2014; Cabanach, Fernández-Cervantes et al., 2010; Cabanach, Valle et al., 2010). También las deficiencias metodológicas del profesorado, las intervenciones en público y la sobrecarga son percibidas habitualmente como amenazantes. Por el contrario, la baja autoestima académica resultó ser la circunstancia que menos preocupa a los universitarios, tal y como ha sido descrito en la literatura existente (Cabanach, Fernández-Cervantes et al., 2010; Cabanach, Valle et al., 2010; Cabanach et al., 2007; Cabanach et al., 2014). Por lo tanto, podría concluirse que los estudiantes perciben como estresantes las situaciones en las que son evaluados (exámenes e intervenciones en público), y también sienten estrés por la excesiva demanda de trabajo y por las deficiencias metodológicas. Teniendo en cuenta que evaluación, carga y metodología son impuestas por el profesor, estos efectos podrían deberse a un sentimiento de escasa autodeterminación o control por parte del estudiante. 
Tabla 6 Análisis de regresión sobre las dimensiones evaluadas

\begin{tabular}{|c|c|c|c|c|c|c|}
\hline & $\mathrm{R}^{2}$ & $\mathrm{~F}$ & $p$ & $\beta$ & $\mathrm{t}$ & $p$ \\
\hline VD: Cansancio & 13,1 & 80,399 & 0,000 & & & \\
\hline Carencia de valor de contenidos & & & & 0,448 & 8,967 & 0,000 \\
\hline VD: Cinismo & 17,1 & 55,205 & 0,000 & & & \\
\hline Carencia de valor de contenidos & & & & 0,527 & 10,385 & 0,000 \\
\hline Exámenes & & & & $-0,157$ & $-3,264$ & 0,001 \\
\hline VD: Eficacia & 7,5 & 9,548 & 0,000 & & & \\
\hline Baja autoestima académica & & & & $-0,214$ & $-3,926$ & 0,000 \\
\hline Deficiencias metodológicas & & & & 0,264 & 3,726 & 0,000 \\
\hline Carencia de valor de contenidos & & & & $-0,199$ & $-3,785$ & 0,000 \\
\hline Falta de participación & & & & 0,143 & 2,737 & 0,006 \\
\hline Intervenciones en público & & & & $-0,075$ & $-2,269$ & 0,024 \\
\hline VD: Rendimiento & 9,5 & 14,303 & 0,000 & & & \\
\hline Intervenciones en público & & & & $-0,148$ & $-4,957$ & 0,000 \\
\hline Deficiencias metodológicas & & & & 0,248 & 4,067 & 0,000 \\
\hline Falta de control sobre el rendimiento & & & & $-0,203$ & $-3,679$ & 0,000 \\
\hline Falta de participación & & & & 0,104 & 2,194 & 0,029 \\
\hline
\end{tabular}

Respecto al burnout, los estudiantes con altos niveles de cansancio presentan también alto cinismo, mientras que aquellos con alta eficacia presentan escaso cansancio y cinismo. En efecto, existe un alto consenso en describir el burnout académico en estos términos (Extremera, Durán y Rey, 2007; Salanova, Martínez, Bresó, Llorens y Grau, 2005; Salanova, Schaufeli, Martínez y Breso, 2010; Schaufeli et al., 2002; Schaufeli y Salanova, 2007).

Asimismo, los estudiantes que se perciben como eficaces presentan un alto rendimiento. En cambio, los que sienten cansancio presentan un rendimiento inferior, mientras que el cinismo no afecta al rendimiento. Consecuentemente, el burnout provoca un deterioro en el rendimiento en tanto en cuanto dicho burnout esté caracterizado por el cansancio (Salanova et al., 2010).

Por otra parte, aunque previamente se ha demostrado que existe una estrecha relación entre estrés percibido y burnout académico (Durán, Extremera, Rey, FernándezBerrocal y Montalbán, 2006; Extremera et al., 2007), hasta el momento no se había determinado cuáles son las situaciones responsables del cansancio, el cinismo o la eficacia.

En el presente estudio se ha encontrado que todas las fuentes de estrés analizadas se asocian con el cansancio y la mayoría de ellas se relacionan también con el cinismo. No obstante, la preocupación por algunas situaciones estresantes se asocia con altos niveles de eficacia académica y de rendimiento (por ejemplo, la falta de participación), mientras que otras se relacionan con bajos niveles de eficacia académica y de rendimiento (por ejemplo, las intervenciones en público).

Por lo que se refiere a los análisis de regresión, se ha puesto de manifiesto la influencia de diferentes situaciones estresantes en el burnout y en el rendimiento.

Concretamente, destaca que la única situación que predice significativamente todas las dimensiones del burnout es la carencia de valor de los contenidos. El estrés debido a la carencia de valor hace referencia a la desazón por estar estudiando contenidos que carecen de interés, provecho o significado práctico. Así, los estudiantes que frecuentemente sienten que los contenidos carecen de utilidad muestran niveles significativamente mayores de cansancio y cinismo, y menores de eficacia.

Si se considera cada dimensión del burnout por separado, se encuentra que la carencia de valor es el único estresor que predice de forma significativa los niveles de cansancio emocional. Por otra parte, el cinismo se predice a partir de la preocupación por la carencia de valor y también por la baja preocupación por los exámenes. Por otra parte, la eficacia se predice a partir de la frecuente preocupación por las deficiencias metodológicas y la falta de participación, además de por una despreocupación por las intervenciones en público, por la baja autoestima académica y por la carencia de valor de contenidos. Estos resultados confirmarían la definición de la eficacia académica entendida como la propia valoración de ser competente para controlar y ejecutar con éxito las actividades académicas (Salanova et al., 2010; Schaufeli et al., 2002; Schaufeli y Salanova, 2007).

Por último, el rendimiento también se predice por la frecuente preocupación por las deficiencias metodológicas y por la falta de participación. Quizá esto pueda deberse a que esta preocupación les lleve a tomar la iniciativa para suplir estas carencias académicas (bien implicándose más ellos mismos en la regulación de sus estudios, bien demandando al profesorado una mejora de las condiciones) y alcanzar así un mejor rendimiento. Recientemente, se ha evidenciado que el rendimiento académico está altamente relacionado con la habilidad de autorregulación académica o participación activa de los universitarios (Gutiérrez-Braojos, SalmerónPérez y Muñoz-Cantero, 2014). Finalmente, la preocupación por las intervenciones en público afecta negativamente al rendimiento, en el mismo sentido que a la eficacia. Por consiguiente, resulta de especial relevancia tratar de reducir el estrés hacia la exposición en público e incrementar las capacidades oratorias de los estudiantes. 
En definitiva, conociendo las fuentes de estrés que afectan al burnout y al rendimiento de los estudiantes, la comunidad educativa debería implicarse en la revisión y la modificación de aquellas situaciones estresantes de las que son responsables (por ejemplo, la metodología empleada, la confianza en la participación de los estudiantes y la relevancia de los contenidos), considerando la opinión de los estudiantes. Una posible medida para dar respuesta a estas necesidades sería adoptar metodologías activas de enseñanza. Un claro ejemplo lo representa el denominado service learning o aprendizaje-servicio, que hace referencia a una herramienta pedagógica que combina el aprendizaje reflexivo y significativo de conocimientos y habilidades que adquieren significado y valor al implementar un servicio a la comunidad (Furco y Billing, 2002; Martínez, 2010). Utilizando esta metodología, los estudiantes participan activamente en la formulación de los objetivos, la planificación del proyecto y su ejecución en la comunidad, observando directamente la utilidad y el significado de los contenidos académicos.

Asimismo, es preciso diseñar estrategias para instruir a los estudiantes en la adquisición de competencias que les permitan afrontar situaciones estresantes. En este sentido, destaca la relevancia del desarrollo de la inteligencia emocional como recurso para hacer frente al estrés académico (Durán et al., 2006). Además, se ha confirmado que los estudiantes con altos niveles de inteligencia emocional presentan bajos niveles de cansancio y cinismo y altos niveles de eficacia (Extremera et al., 2007). Sustancialmente, se trataría de diseñar programas con el objetivo de enseñar a los estudiantes a percibir, diferenciar, comprender y regular sus emociones para hacer frente a las circunstancias estresantes de forma eficaz.

Alternativamente, en los últimos años ha cobrado notoriedad el empleo de técnicas de meditación para prevenir y combatir el estrés y el burnout en universitarios (Franco, 2009; Gallego, Aguilar-Parra, Cangas, Rosado y Langer, 2016). Desde esta perspectiva, se defiende que mantener la conciencia en el presente previene la aparición de pensamientos obsesivos, relacionados con el cansancio emocional, que limitan la capacidad de concentración del estudiante. Por ello, debería promoverse la práctica de la meditación entre los universitarios para tratar de mejorar su bienestar.

Por último, también convendría desarrollar iniciativas para mejorar las competencias de los estudiantes para hablar en público. En esta línea, recientemente se ha propuesto un programa de instrucción en habilidades para realizar presentaciones orales (García-López, Díez-Bedmar y Almansa-Moreno, 2013). Concretamente, se entrena a los estudiantes en comunicación verbal y no verbal (contacto ocular, gestos, entonación, etc.), consiguiendo así mitigar el estrés hacia estas actuaciones.

En suma, mediante estas medidas se conseguiría moderar los niveles de estrés en los estudiantes, reducir su cansancio y cinismo, fortalecer su autoeficacia y mejorar su rendimiento.

Como limitaciones del estudio debe señalarse que se ha evaluado a estudiantes de 3 grados distintos: Educación Infantil, Educación Social y RRLL y RRHH, y teniendo en cuenta que existen diferencias entre titulaciones en la percepción de estresores (Cabanach, Fernández-Cervantes et al., 2010), en futuras investigaciones debería incluirse la mayor variedad de grados posible. Otra limitación a destacar es que la mayoría de los estudiantes evaluados son mujeres, y dado que pueden existir diferencias en función del género en relación con el rendimiento y el burnout (Martínez y Salanova, 2003), es necesario que en futuros trabajos se equipare el número de hombre y mujeres, lo cual probablemente quede subsanado al ampliar el rango de grados evaluados.

\section{Bibliografía}

Arribas, J. (2013). Hacia un modelo causal de las dimensiones del estrés académico en estudiantes de Enfermería. Revista de Educación, 360, 533-556. http://dx.doi.org/10.4438/ 1988-592X-RE-2011-360-126

Bresó, E., Schaufeli, W. B. y Salanova, M. (2011). Can a selfefficacy-based intervention decrease burnout, increase engagement, and enhance performance? A quasi-experimental study. Higher Education, 61, 339-355. http://dx.doi.org/10.1007/ s10734-010-9334-6

Cabanach, R. G., Fernández-Cervantes, R., González-Doniz, L. y Freire, C. (2010). Estresores académicos percibidos por estudiantes universitarios de ciencias de la salud. Fisioterapia, 32, 151-158. http://dx.doi.org/10.1016/j.ft.2010.01.005

Cabanach, R. G., Rodríguez, S., Valle, A., Piñeiro, I. y Millán, P. G. (2007). Metas académicas y vulnerabilidad al estrés en contextos académicos. Aula Abierta, 36, 3-16.

Cabanach, R. G., Souto, A., Freire, C. y Ferradás, M. M. (2014). Relaciones entre autoestima y estresores percibidos en estudiantes universitarios. European Journal of Education and Psychology, 7, 43-57. http://dx.doi.org/10.1989/ejep.v7i1.151

Cabanach, R. G., Valle, A., Rodríguez, S. y Piñeiro, I. (2008). Respuesta de estrés en contextos universitarios: construcción de una escala de medida. V Congreso Internacional de Psicología y Educación: los retos de futuro. Oviedo.

Cabanach, R. G., Valle, A., Rodríguez, S., Piñeiro, I. y González, P. (2010). Las creencias motivacionales como factor protector del estrés en estudiantes universitarios. European Journal of Education and Psychology, 3, 75-87. http://dx.doi.org/10.1989/ejep.v3i1.47

Chambel, M. J. y Curral, L. (2005). Stress in academic life: Work characteristics as predictors of student well-being and performance. Applied Psychology: An International Review, 54, 135-147. http://dx.doi.org/10.1111/j.1464-0597.2005.00200.x

Chemers, M. M., Hu, L. T. y Garcia, B. F. (2001). Academic self-efficacy and first year college student performance and adjustment. Journal of Educational Psychology, 93, 55-64. http://dx.doi.org/10.1037/0022-0663.93.1.55

Durán, A., Extremera, N. E., Rey, L., Fernández-Berrocal, P. y Montalbán, F. M. (2006). Predicting academic burnout and engagement in educational settings: Assessing the incremental validity of perceived emotional intelligence beyond perceived stress and general self-efficacy. Psicothema, 18, 158-164.

Extremera, N. E., Durán, A. y Rey, L. (2007). Inteligencia emocional y su relación con los niveles de burnout, engagement y estrés en estudiantes universitarios. Revista de Educación, 342, 239-256. http://dx.doi.org/ 10.4438/1988-592X-RE-2011-359-109

Franco, C. (2009). Modificación de los niveles de burnout y de personalidad resistente en un grupo de deportistas a través de un programa de conciencia plena (mindfulness). Anuario de Psicología, 40, 377-390.

Freudenberger, H. J. (1974). Staff burnout. Journal of Social Issues, 30, 159-165. http://dx.doi.org/10.1111/j.1540-4560. 1974.tb00706.x 
Furco, A. y Billing, S. H. (2002). Service-learning. The essence of the pedagogy. Greenwich, Connecticut: Information Age Publishing Inc.

Gallego, J., Aguilar-Parra, J. M., Cangas, A. J., Rosado, A. y Langer, A. I. (2016). Efecto de intervenciones mente/cuerpo sobre los niveles de ansiedad, estrés y depresión en futuros docentes de educación primaria: Un estudio controlado. Revista de Psicodidáctica, 21, 87-101. http://dx.doi.org/ 10.1387/RevPsicodidact.13256

García-López, L. J., Díez-Bedmar, M. B. y Almansa-Moreno, J. M. (2013). De ser entrenado a ser entrenador: cómo ayudar a otros iguales en la mejora de las competencias para hablar en público. Revista de Psicodidáctica, 18, 331-342. http://dx.doi.org/10.1387/RevPsicodidact.6419

Gratacós, G. y López-Jurado, M. (2016). Validación de la versión en español de la escala de los factores que influyen en la elección de los estudios de educación (FIT-choice). Revista de Educación, 372, 87-110. http://dx.doi.org/10.4438/ 1988-592X-RE-2015-372-316

Gutiérrez-Braojos, C., Salmerón-Pérez, H. y Muñoz-Cantero, J. M. (2014). El efecto modulador de los patrones temporales sobre el logro en el aprendizaje autorregulado. Revista de Psicodidáctica, 19, 267-287. http://dx.doi.org/10.1387/ RevPsicodidact.10066

Kanner, A. D., Coyne, J. C., Schaefer, C. y Lazarus, R. S. (1981). Comparison of two modes of stress measurement: Daily hassles and uplifts versus major life events. Journal of Behavioral Medicine, 4, 1-39.

Lazarus, R. S. (2000). Estrés y emoción. Manejo e implicaciones en nuestra salud. Bilbao: Desclée de Brouwer.

Manzanares, D. y Martínez, S. (2014). Rúbrica y evaluación recíproca en el trabajo de grupo en la asignatura Historia de las Relaciones Laborales. Trabajo, 30, 137-161.

Manzano, G. (2002). Burnout y engagement. Relación con el desempeño, madurez profesional y tendencia al abandono de los estudiantes. Revista de Psicología Social, 17, 237-249. http://dx.doi.org/10.1174/02134740260372973

Manzano, G. (2004). Perfil de los estudiantes comprometidos con sus estudios: influencia del burnout y el engagement. Anuario de Psicología, 35, 399-415.

Martín, I. M. (2007). Estrés académico en estudiantes universitarios. Apuntes de Psicología, 25, 87-99.
Martínez, M. (2010). Aprendizaje servicio y responsabilidad social de las universidades. Barcelona: Octaedro.

Martínez, I., Marques, A., Salanova, M. y Lopez da Silva, A. (2002). Burnout en estudiantes universitarios de España y Portugal. Ansiedad y Estrés, 8, 13-23.

Martínez, I. y Salanova, M. (2003). Niveles de burnout y engagement en estudiantes universitarios. Relación con el desempeño y desarrollo profesional. Revista de Educación, 330, 361-384.

Maslach, C. (1976). Burned-out. Human Behavior, 9, 16-22.

Maslach, C., Schaufeli, W. B. y Leiter, M. P. (2001). Job burnout. Annual Review of Psychology, 52, 397-422.

Mendo-Lázaro, S., León, B., Felipe-Castaño, E., Polo, M. I. y Palacios-García, V. (2016). Evaluación de las habilidades sociales de estudiantes de Educación Social. Revista de Psicodidáctica, 21, 139-156. http://dx.doi.org/10.1387/RevPsicodidact.14031

Muñoz, F. J. (2004). El estrés académico. Problemas y soluciones desde una perspectiva psicosocial. Huelva: Servicio de Publicaciones, Universidad de Huelva.

Peñacoba, C. y Moreno, B. (1999). La escala de estresores universitarios (EEU). Una propuesta para la evaluación del estrés en grupos poblacionales específicos. Ansiedad y Estrés, 5, 61-78.

Salanova, M., Bresó, E. y Schaufeli, W. B. (2005). Hacia un modelo espiral de las creencias de eficacia en el estudio del burnout y del engagement. Ansiedad y Estrés, 11, 215-231.

Salanova, M., Martínez, I. M., Bresó, E., Llorens, S. y Grau, R. (2005). Bienestar psicológico en estudiantes universitarios: facilitadores y obstaculizadores del desempeño académico. Anales de Psicología, 21, 170-180.

Salanova, M., Schaufeli, W., Martínez, I. y Breso, E. (2010). How obstacles and facilitators predict academic performance: The mediating role of study burnout and engagement. Anxiety, Stress and Coping, 23, 53-70. http://dx.doi.org/10.1080/ 10615800802609965

Schaufeli, W. B., Martínez, I., Marqués-Pinto, A., Salanova, M. y Bakker, A. (2002). Burnout and engagement in university students: A cross-national study. Journal of Cross-Cultural Studies, 33, 464-481.

Schaufeli, W. B. y Salanova, M. (2007). Efficacy or inefficacy, that's the question: Burnout and work engagement, and their relationships with efficacy believes. Anxiety, Stress and Coping, 20, 177-196. http://dx.doi.org/10.1080/10615800701217878 